\title{
Rare Case of Spontaneous Rectus Sheath Hematoma with Intraperitoneal Extension in Dengue Hemorrhagic Fever: A Case Report
}

\author{
Mahesh $^{1}$, Rithi Melissa D Silva ${ }^{2}$, MD Kaleemullah $^{3}$, Prajnha U P4 \\ ${ }^{1}$ Senior Resident, Department of Radio Diagnosis, ${ }^{2} J u n i o r$ Resident, Department of Radio Diagnosis, 3Junior Resident, \\ Department of Radio Diagnosis, ${ }^{4} \mathrm{~K}$ S Hegde Institute of Medical Scienes, Deralakatte, Mangalore India
}

Corresponding author: Mahesh, Senior Resident, Department of Radio Diagnosis, Father Muller Medical College and Hospital, Kankanady, Managaluru-575002, India

DOI: http://dx.doi.org/10.21276/ijcmsr.2019.4.1.1

How to cite this article: Mahesh, Rithi Melissa D Silva, MD Kaleemullah, Prajnha U P. Rare case of spontaneous rectus sheath hematoma with intraperitoneal extension in dengue hemorrhagic fever: a case report. International Journal of Contemporary Medicine Surgery and Radiology. 2019;4(1):A1-A3.

\section{A B S T R A C T}

Introduction: Muscle hematomas are a rare complication of dengue fever and only a handful of cases have been reported in literature. It needs to be promptly identified for suitable management.

Case report: We report a case of 63-year-old-female admitted with dengue fever who developed spontaneous rectus sheath hematoma. The findings were confirmed by abdominal ultrasonography and magnetic resonance imaging.

Conclusion: In conclusion, the clinicians must be vigilant in the convalescent phase of dengue fever for manifestations like spontaneous retroperitoneal and intramuscular haematomas which like haemorrhages in other major organs or body cavities need prompt diagnosis and management

Key words: Dengue Haemorrhagic Fever, Rectus Sheath Hematoma

\section{INTRODUCTION}

Dengue virus is a vector single-stranded RNA virus belonging to flavivirus genus. ${ }^{1,2}$ Dengue infection causes dengue fever (DF), dengue hemorrhagic fever (DHF), and dengue shock syndrome (DSS). ${ }^{3}$

DHF commonly manifests as superficial bleeding (e.g. ecchymosis, petechiae, gum bleeding. ${ }^{4}$ Gastrointestinal and intracranial haemorrhage may occur rarely in DHF

The pathogenesis of bleeding in dengue fever is attributed to hyperimmune response of the host to the dengue virus which causes endothelial dysfunction, increased vascular permeability and thrombocytopenia with or without platelet dysfunction. ${ }^{5}$ There is only little correlation between haematological changes with clinical bleeding. ${ }^{6}$

Rectus sheath hematoma $(\mathrm{RSH})$ is a rare occurrence in $\mathrm{DHF}$ and is characterized by bleeding within the rectus abdominis muscle sheath due to ruptured vessels or a tear of muscle. ${ }^{7}$ Only three cases of spontaneous RSH in DHF have been described in literature. ${ }^{8}$ Some known risk factors of spontaneous $\mathrm{RSH}$ are pregnancy, obesity, female gender, anticoagulants, abdominal straining, severe vomiting, and persistent cough.

\section{CASE REPORT}

A 63-year-old female presented to the medicine outpatient department with fever, nausea, vomiting, generalised body pain along with an episode of giddiness and fall. She also gave history of passing black coloured stools. On arrival, examination of the patient revealed stable vitals with pulse: $78 \mathrm{bpm}$, blood pressure: 130/80, respiratory rate: 18 breaths / min. Her body temperature was $39{ }^{\circ} \mathrm{C}$. Respiratory, cardiovascular system and central nervous system examinations were unremarkable. Routine chest $\mathrm{x}$ ray also revealed no significant abnormality. Following admission, her lab investigations revealed haemoglobin of $12 \mathrm{~g} / \mathrm{dl}$, total counts of $2800 /$ cumm (leucopaenia), platelet count 69,000 (thrombocytopenia). Further, dengue rapid NS1 and anti Dengue IgM test was positive. Serum electrolytes, urine analysis, liver function tests were unremarkable.

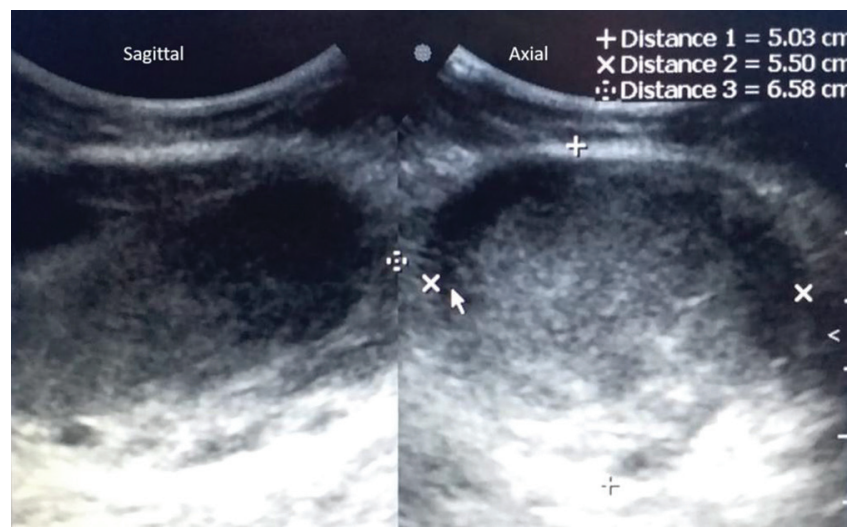

Figure-1: USG images showing a hypoechoic left rectus sheath hematoma 


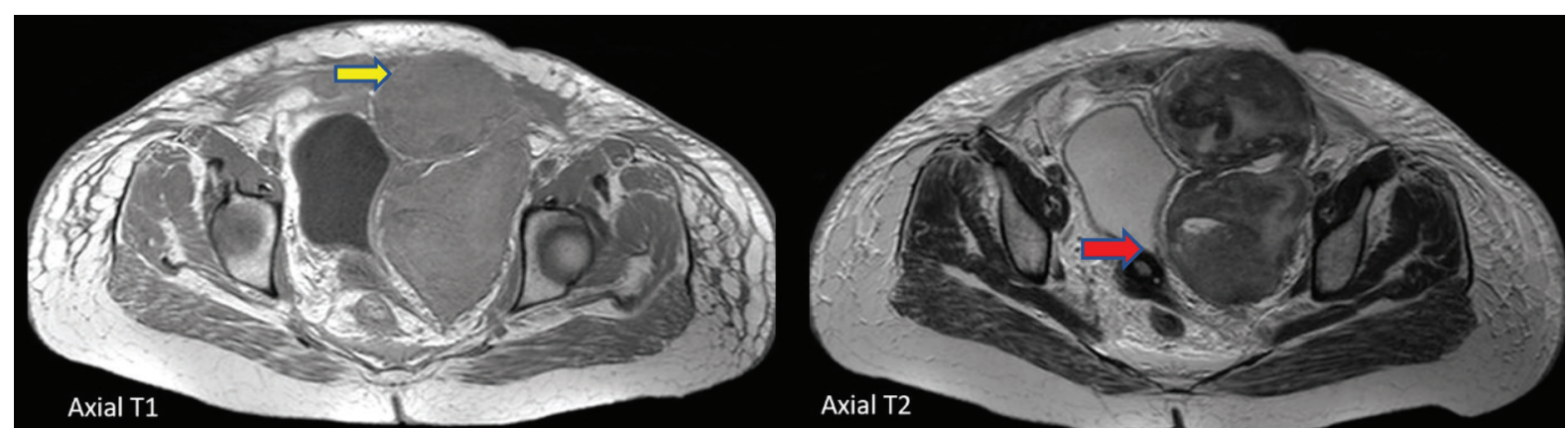

Figure-2: Axial MRI images showing a T1 iso to mildly hyperintense and T2 heterogenously hypointense left rectus sheath hematoma (yellow arrow) with intraperitoneal component causing mass effect on bladder (red arrow).

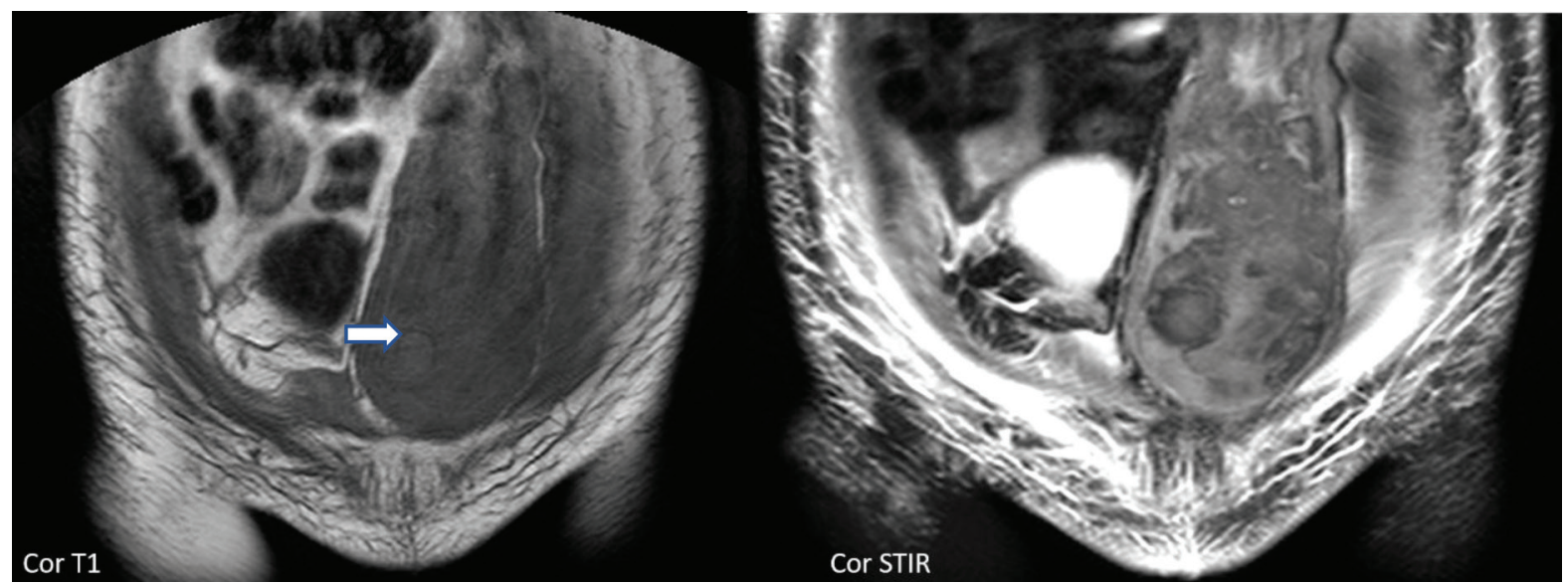

Figure-3: Coronal T1 and STIR sequences showing the hematoma along the left rectus sheath (arrow)

Renal function test revealed an elevated creatinine (1.6).

On day 5, of admission, patient complained of sudden severe left lower abdomen pain. On examination, there was a palpable mass in left lower iliac region. This was evaluated by ultrasound abdomen, which showed a well-defined ovoid heterogeneously hypoechoic lesion measuring $50 \times 55 \times 65$ $\mathrm{mm}$ in left lower rectus consistent with hematoma(Fig 1). Lab tests revealed a drop in the haemoglobin level to $9 \mathrm{~g} / \mathrm{dl}$ and reduction in platelet counts to 20,000.

When the patients symptoms worsened on day 6 and 7,MRI of abdomen and pelvis was done to confirm the findings and to rule out other causes. Contrast $\mathrm{CT}$ was avoided in view of the deranged renal function tests. MRI showed large rectus sheath hematoma in left lower abdomen measuring $83 \times 66$ $\mathrm{x} 181 \mathrm{~mm}$ and also interval development of intraperitoneal extension of the hematoma measuring $80 \times 70 \times 86 \mathrm{~mm}$ causing displacement of pelvic structures to right side (Fig 2 and Fig3)

Surgery reference for the same was sought and the patient was treated conservatively with packed cell transfusions and intravenous antibiotics. Serial ultrasound examinations were conducted in the following week to measure the size of hematoma, which showed gradual resolution.

The platelet counts improved with platelet transfusions during the course of stay and gradual increase in platelet counts to up to $1,76,000 / \mathrm{cu} \mathrm{mm}$ was noted on day 19 of at the time of discharge.

Patient's clinical condition improved over the course in the hospital and was she discharged with stable vitals.
DISCUSSION

The pathogenesis of bleeding in dengue fever is attributed to hyperimmune response of the host to the dengue virus. This results in endothelial dysfunction, altered procoagulation, fibrinolysis, vascular fragility and thrombocytopenia with or without platelet dysfunction. ${ }^{5,9}$

The critical stage of DHF carries the risk of circulatory failure and bleeding complications. ${ }^{10}$ Muscles hematomas are very rare complications of $\mathrm{DHF}^{11}$ The common sites of intramuscular hematomas in the abdomen are the rectus sheath, psoas and iliac muscles. Although it is selflimiting it can turn into a hemoperitoneum as in the case described. ${ }^{12}$ These patients present with palpable abdominal mass associated with pain. The imaging modalities useful in the diagnosis of RSH are ultrasonography (USG), CTscan, and MRI. USG is the initial procedure of choice because of its low cost, high sensitivity rates absence of risk of radiation. Among the cross sectional modalities, MRI is more superior than CT estimation of age of the hematoma and in exact anatomical localization. Treatment of $\mathrm{RSH}$ is mainly intravenous hydration and blood transfusion. Surgical intervention is advised only when conservative management fails

\section{CONCLUSION}

In conclusion, the clinicians must be vigilant in the convalescent phase of dengue fever for manifestations like spontaneous retroperitoneal and intramuscular haematomas which like haemorrhages in other major organs or body cavities need prompt diagnosis and management. 


\section{REFERENCES}

1. Jayasinghe NS, Thalagala E, Wattegama M, Thirumavalavan K. BMC Res Notes. 2016; 9(3):265.

2. Rodenhuis-Zybert IA, Wilschut J, Smit JM. Dengue virus life cycle: viral and host factors modulating infectivity. Cell Mol Life Sci. 2010;67(6):2773-86.

3. Mahale R, Mehta A, Srinivasa R. J Neurosci Rural Pract. 2016; 7(2): 323-324.

4. Kalayanarooj S. Clinical manifestations and management of Dengue/DHF/DSS. Trop Med Health. 2011;39(Suppl 4):83-87.

5. Ganu S,Mehta Y.Femoral compressive neuropathy from iliopsoas haematoma complicating dengue haemorrhagic fever. Asian Pac J Trop Med. 2013;6(5):419- 20.

6. De Azeredo E.L., Monteiro R.Q., de- Oliveira Pinto L.M. Thrombocytopenia in dengue: interrelationship between virus and the imbalance between coagulation and fibrinolysis and inflammatory mediators. Mediat Inflamm. 2015;2015:313842.

7. Bhat K.J, Shovkat R, Samoon H.J. Abdominal haematomas and dengue fever: two different cases of spontaneous psoas muscle haematoma and bilateral rectus sheath haematoma complicating dengue haemorrhagic fever.J Vector Borne Dis. 2015;52(4):339_ 341.

8. Kaur H, Girgila K. Rectus sheath haematoma complicating dengue haemorrhagic fever a case report. Int J Res Med Sci 2017;5(4):354-6.

9. Wills BA, Oragui EE, Stephens AC, Daramola OA, Dung NM, Loan HT, et al.Coagulation abnormalities in dengue hemorrhagic fever: serial investigations in 167 Vietnamese children with dengue shock syndrome. Clin Infect Dis 2002;35(3):277-85

10. Martina BE, Koraka P, Osterhaus AD. Dengue virus pathogenesis: an integrated view. Clin Microbiol Rev 2009;22(4):564-81.

11. Nelwana EJ, Angelinaa F, Adiwinataa R,Matondangb S, Andrionoc P. Spontaneous rectus sheath hematomas in dengue hemorrhagic fever: A case report. IDCases. 2017; 10(1): 35-37.

12. Cherry WB, Mueller PS. Rectus sheath hematoma: review of 126 cases at a single institution. Medicine (Baltimore) 2006;85(2):105-10.

\section{Source of Support: Nil; Conflict of Interest: None}

Submitted: 09-12-2018; Accepted: 11-01-2019; Published online: 21-01-2019 